\title{
UTILIZAÇÃO DE TERAPIAS INTEGRATIVAS E COMPLEMENTARES EM UNIDADES DE TERAPIA INTENSIVA: REVISÃO INTEGRATIVA DA LITERATURA
}

Use of integrative and complementary therapies in intensive care units: integrative review of literature

\author{
Karina Vilela dos Santos ${ }^{1}$; João Ricardhis Saturnino de Oliveira ${ }^{2}$
}

${ }^{1}$ Fisioterapeuta (UFPE). Pós-Graduada em Terapia Intensiva (CEFAPP/FACESF). Residente em Nefrologia (PRMIS/HC-UFPE).E-mail: karinavilelas@hotmail.com

${ }^{2}$ Fisioterapeuta e Mestre em Bioquímica e Fisiologia (UFPE).

Data do recebimento: 18/10/2019 - Data do aceite: 28/02/2020

RESUMO: Trata-se de pesquisa descritiva de caráter qualitativo realizada a partir da busca na literatura de evidências dos benefícios de terapias complementares e integrativas na redução de sintomas, como dor e constipação, durante o internamento em unidade de terapia intensiva. Os estudos utilizados foram publicados entre 2008 e 2018, em unidades para adultos, pediátricas e neonatal. De uma forma geral, todos os trabalhos encontraram benefícios nas técnicas utilizadas, sendo a massagem e a musicoterapia as mais estudadas. Como benefício, a massagem reduziu quadros álgicos, ansiedade de pacientes pós-cirúrgicos, bem como melhorou a motilidade gastrointestinal. A musicoterapia, ao vivo e a partir de músicas para relaxamento num fone de ouvido, reduziu a ansiedade de pacientes entubados, assim como melhorou a qualidade do sono. Assim, foram levantadas algumas das técnicas complementares e integrativas que podem ser utilizadas por fisioterapeutas para melhorar a qualidade de vida de pacientes durante o internamento em unidades de terapia intensiva.

Palavras-chave: Fisioterapia. Unidade de Terapia Intensiva. Terapias Complementares e Integrativas.

ABSTRACT: This is a qualitative descriptive research carried out based on the literature search for evidence of the benefits of complementary and integrative 
therapies in reducing symptoms, such as pain and constipation, during hospitalization in an intensive care unit. The studies used were published between 2008 and 2018, in adult, pediatric and neonatal units. In general, all the studies found benefits in the techniques used, with massage and music therapy as the most studied. As a benefit, massage has reduced pain, post-surgical anxiety, as well as improved gastrointestinal motility. Music therapy, live and from recorded songs for relaxation in a headset, reduced the anxiety of intubated patients, as well as improved sleep quality. Thus, some complementary and integrative techniques, which can be used by physical therapists to improve the quality of life of patients during hospitalization in intensive care units, were raised.

Keywords: Physiotherapy. Intensive Care Unit. Complementary and Integrative Therapies.

\section{Introdução}

O ambiente hospitalar é considerado o melhor lugar para atenção e cuidado de pacientes internados. No Brasil, cerca de 3,5 milhões de indivíduos são internados para realização de novos diagnósticos e acompanhamento de patologias crônicas. Em alguns casos, necessidade de cuidados intensivos, sendo então admitidos em Unidade de Terapia Intensiva (UTI) (BRASIL, 2010; MIOKOVIC et al., 2014; JESUS et al., 2016).

A rotina de uma UTI é, em grande parte, voltada para intervenções de emergência, na tentativa de estabilização do quadro do paciente, sendo frequentemente necessária a utilização de medicamentos para estabilizar os sinais vitais, como pressão arterial e frequência cardíaca. Diante da cronicidade das internações, média de 11 dias, os pacientes necessitam de outros cuidados, como a inserção de medicamentos analgésicos e ansiolíticos, por exemplo, além de assistência para a movimentação do corpo (BRASIL, 2010; HU et al., 2015).

O cenário da UTI também contribui como fator agravante para as comorbidades do indivíduo, visto que, durante o período de internação, os pacientes se deparam com um ambiente estressante, de grande ruído, volumosa equipe profissional e elevada demanda de procedimentos. Neste contexto, a rotina do setor pode promover o estresse e prejuízo ao sono, além de favorecer a instalação de quadros de ansiedade, depressão e delirium, contribuindo com a piora da qualidade de vida e gerando risco de traumas pós-internamento (MISTRALETTI et al., 2008; ORWELIUS et al., 2008; HU et al., 2015).

$\mathrm{Na}$ intenção de melhorar a qualidade de vida de pacientes internados em UTI, surgiu o conceito de humanização da assistência, na qual se preza pelo cuidado e respeito ao tempo e espaço de cada indivíduo, bem como preconiza uma assistência menos técnica e mecanicista, valorizando o toque e a interação da equipe multiprofissional de saúde com o paciente. O profissional de saúde passa a trabalhar mais próximo do paciente, tentando atender às suas necessidades individuais $\mathrm{e}$ subjetivas. Neste contexto, o fisioterapeuta, enquanto integrante desta equipe, é de suma importância (ORWELIUS et al., 2008; FUENTE-MARTOS et al., 2018).

Apesar de várias técnicas se mostrarem eficazes no tratamento dos pacientes 
admitidos na UTI, existem ainda aqueles indivíduos que estão em seu segundo ou terceiro internamento, que convivem com suas patologias há décadas, o que favorece a uma maior dificuldade de resposta às técnicas convencionais. Desta forma, as técnicas consideradas complementares e integrativas vêm sendo cada vez mais utilizadas, estando ligadas à outras formas de visualização do processo saúde-doença, como na visão oriental (MISTRALETTI et al., 2008; BRASIL, 2010; FUENTES-MARTOS et al., 2018).

A partir de uma visão global do paciente, e de suas necessidades como ser vivo, as técnicas complementares, muitas vezes, trabalham a melhor movimentação das energias corporais, conhecidas como Qi (qui), ou outras técnicas não convencionais, que vêm mostrando cada vez mais benefícios para pacientes que não conseguem mais obter bons resultados com tratamentos farmacológicos e técnicas tradicionais da medicina ocidental (NASCIMENTO et al., 2012).

Entretanto, mesmo sabendo dos benefícios que estas técnicas podem trazer, muitos profissionais se recusam a utilizálas, principalmente por não conhecerem, mas também por acharem que tais técnicas não podem ser agregadas ao seu leque de ferramentas durante as condutas (HARVEY-WALKER, 1998; NASCIMENTO et al., 2012). Outro conflito em relação à aplicação das técnicas complementares e integrativas vem dos demais membros da equipe, devido ao elevado risco de mortalidade destes pacientes em detrimento da condição clínica estabelecida, visto que muitos dos pacientes se encontram em estado grave de infecção ou de instabilidade hemodinâmica (WANG et al., 2010).

Diante deste contexto, o fisioterapeuta se mostra aberto para a incorporação dessas técnicas, sobretudo devido a muitas delas lidarem com o toque e o reequilíbrio mental e corporal do paciente (HARVEY-WALKER, 1998; NASCIMENTO et al., 2012). Desta forma, estudos que validem a aplicação de técnicas complementares e integrativas, bem como suas utilizações pelo fisioterapeuta, são necessários para sua incorporação segura nas condutas da UTI.

\section{Métodos}

Trata-se de uma revisão integrativa da literatura, cuja busca por artigos foi realizada por dois pesquisadores, de forma individualizada, nas seguintes bases de dados e repositórios: SciELO, PubMed, Cochrane Library e Biblioteca Virtual de Saúde. Foram utilizadas as palavras-chave e descritores: Fisioterapia, Terapia Complementar, Terapia Integrativa, Medicina Alternativa, Unidade de Terapia Intensiva e Fisioterapia Intensiva. Os termos foram utilizados na língua portuguesa, mas também em seus correspondentes em inglês e espanhol. Foram aceitos trabalhos completos, realizados com humanos, publicados entre 2000 e 2019, que foram realizados ou sugeridos para realização por fisioterapeutas. Também foi tomado em consideração que estes trabalhos avaliassem benefícios das terapias sobre dor, ansiedade, qualidade de vida e tempo de internamento. Foram excluídos trabalhos realizados em outros ambientes hospitalares além de Unidades de Terapia Intensiva.

\section{Resultados}

A busca bibliográfica obteve 452 artigos, uma grande quantidade. No entanto, após análise dos critérios de inclusão e exclusão, sete artigos foram selecionados para a discussão sobre o uso de técnicas complementares e integrativas na UTI por fisioterapeutas (Figura 1). 
Figura 1 - Estratégia de revisão integrativa da literatura utilizada para seleção de artigos com terapias complementares e integrativas em unidades de terapia intensiva
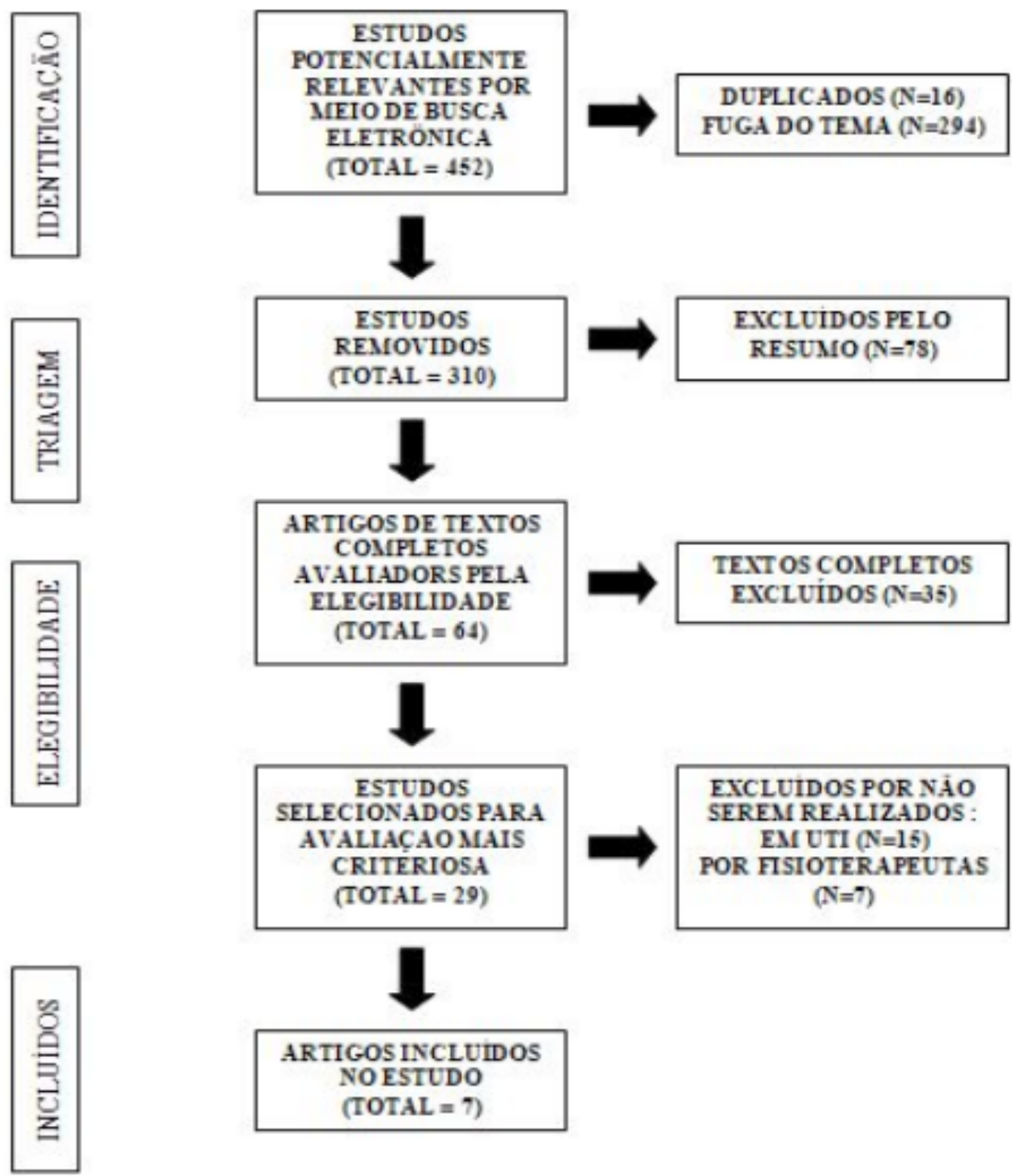

$$
\begin{aligned}
& \text { ARTIGOS INCLUIDOS } \\
& \text { NOESTUDO } \\
& (\text { TOTAL }=7)
\end{aligned}
$$

Dos estudos selecionados, a maior parte utilizou-se de massagem terapêutica. A segunda técnica mais utilizada foi a musicoterapia, como mostra a Tabela I. 
UTILIZAÇÃO DE TERAPIAS INTEGRATIVAS E COMPLEMENTARES EM UNIDADES DE TERAPIA INTENSIVA: REVISÃO INTEGRATIVA DA LITERATURA

Tabela I - Principais informações dos estudos sobre terapias integrativas e complementares realizadas por fisioterapeutas nas Unidades de Terapia Intensiva

\section{Autor e Tipo de Estudo \\ População \\ Intervenção \\ Benefícios}

DEHGHAN et al., 2018

Ensaio Clínico Randomizado

CHO et al., 2013

Ensaio Clínico

CHIASSON et al, 2013

Caso Controle

SILVA et al., 2017

Ensaio Clínico

SAADATMANT et al., 2015

Ensaio Clínico Randomizado

MORAN et al., 2015

Ensaio Clínico Randomizado

MENDES; PROCIANOY, 2008

Ensaio Clínico Randomizado
35 adultos, intubados, recebendo alimentação por sonda nasogástrica.

18 homens e 10 mulheres, 20 pacientes tinham mais de 60 anos, com doença cardíaca isquêmica pós-implante de stent arterial.

26 homens e 24 mulheres com média de 65,29 anos, admitidos na UTI de um hospital universitário.

18 homens e 30 mulheres, com média de 55,46 anos, internados em UTI adulto, com ECGlasgow

$>13$.

30 adultos, internados em UTI, sob ventilação mecânica.

Recém-nascidos com média de $1268 \mathrm{~g}$ ao nascimento e idade gestacional de 31 semanas, internados na UTI Neonatal.

52 recém-nascidos, com aproximadamente $1629,4 \mathrm{~g}$ ao nascer, internados na UTI Neonatal.
15 min de Massagem Suíça na região abdominal, $2 x$ ao dia 30 min antes da alimentação.

Aromaterapia: 10 inspirações em pedras aromáticas contendo duas gotas de mistura de óleos essenciais de lavanda, camomila romana e néroli $(6: 2: 0,5)$.

10 min de toque de Harpa dentro da UTI.

Redução de dor.

30 min de Massagem suíça em membros e região do trapézio.

Redução de ansiedade.

90 min de musicoterapia com fones de ouvido.

Redução de dor.

Musicoterapia com gravador na incubadora $3 \mathrm{~min}$ antes, 15 min durante e 3 min após fisioterapia respiratória.

Melhora da

frequência

respiratória.

\section{Massagem terapêutica} (exercícios cutâneos e exercícios passivos) materna, $4 x / d i a$, com intervalo de $6 \mathrm{~h}$ por $15 \mathrm{~min}$.
Redução do internamento hospitalar $\mathrm{e}$ incidência de sepse tardia em RNBP.
Redução do volume residual, da constipação e da circunferência abdominal.

Redução da ansiedade e melhora da qualidade do sono.

UTI: Unidade de Terapia Intensiva; ECGlasgow: Escala de Coma de Glasgow; RNBP: recém-nascido de baixo peso. 
Apesar de pouco pesquisada, a utilização de técnicas complementares foi avaliada por estudos de qualidade: ensaios clínicos randomizados, com critérios metodológicos bem delimitados. Esta revisão não teve por objetivo avaliar o grau de evidência dos estudos abordados, porém, vale salientar que apenas 2 receberam menos de 4 pontos na escala de qualidade de evidência da base de dados PEDro.

\section{Discussão}

O ensaio clínico randomizado de Dehghan et al. (2018), realizado em 2017, teve como objetivo avaliar os efeitos da massagem abdominal em pacientes internados na UTI, em ventilação mecânica (VM), com nutrição por sonda enteral. Fizeram parte do estudo 70 pacientes em terapia intensiva, dos quais, após serem alocados aleatoriamente, nos grupos de intervenção ou controle, receberam massagem abdominal duas vezes por dia, durante três dias, com duração de 15 minutos cada, ou receberam apenas intervenção de rotina, respectivamente. Foram avaliados os seguintes parâmetros e obtiveram os seguintes resultados: volume residual gástrico diminuído no grupo que sofreu intervenção e aumento no grupo-controle, mesmo assim não houve diferença significativa entre os grupos; no grupo que sofreu intervenção, houve diminuição significativa da circunferência abdominal, tempo de defecação e a frequência de constipação, a prevalência da constipação também diminuiu significativamente.

Sendo assim, os autores indicam o uso da massagem abdominal nos pacientes em $\mathrm{VM}$, com sonda enteral, como recurso não medicinal que traz pouco ou nenhum efeito colateral, diferente dos meios medicinais e da permanência/imobilidade no leito, como mostrados em outros estudos, que trazem repercussões negativas ao sistema gastrintestinal.

Ademais, o estudo de Silva et al. (2017) acrescentou aos benefícios de um tratamento com massagem suíça a redução da dor e da ansiedade de pacientes adultos em UTI com Escala de Coma de Glasgow maior que 13 pontos. Vale salientar que a massagem era realizada principalmente em região de músculo trapézio, e apenas uma vez ao dia. Isto mostra benefícios de uma técnica não farmacológica no combate a sintomas oriundos da cronicidade dos internamentos em UTI.

A música é um recurso que vem sendo largamente utilizado para diminuição de parâmetros fisiológicos em pacientes que sofreram ou estão na iminência de intervenção cirúrgica. É possível verificar isso em estudos, como o de Chiasson et al. (2013). Baseados nos resultados positivos de outros estudos feitos em diferentes contextos, mas com basicamente o mesmo propósito, de correlacionar os efeitos deletérios da permanência em ambiente hospitalar e a música como forma de terapia não farmacológica, usaram a Harpa ao vivo por 10 minutos nos pacientes da UTI (com semelhança na gravidade da doença), em pré ou pós-operatório, aleatoriamente designados, a fim de afirmar suas repercussões na frequência cardíaca (FC), pressão arterial (PA), dor e na sensação de relaxamento. Assim, puderam concluir que houve diminuição da percepção da dor, levando consequentemente à diminuição no uso de opioides, porém os outros parâmetros avaliados não foram significativamente afetados.

Corroborando com Chiasson et al. (2013), o ensaio clínico randomizado realizado por Moran et al. (2014), cujo objetivo foi correlacionar os efeitos da música e os sinais vitais dos recém-nascidos $(\mathrm{RN})$ prematuros sujeitos à fisioterapia respiratória, no qual a amostra foi composta por RN prematu- 
ros, 12 incluídos no grupo-controle e 14 no grupo de intervenção, sendo a intervenção constituída por exposição à música clássica (gravador inserido na incubadora), por 3 minutos antes e após a intervenção fisioterapêutica convencional (15 min - vibração e aspiração), totalizando 21 minutos, sendo medidas as frequências respiratórias (FR), FC e saturação periférica de oxigênio (SpO2) antes, durante e após as intervenções. Como resultado, a FR dos neonatos teve diminuição significativa, mantendo-se em valores basais.

Baseado na afirmação do alívio da dor por meio de manejo não farmacológico, Saadamant et al. (2015) investigaram, por meio de um ensaio clínico randomizado, a aplicação de sons naturais no propósito de reduzir efeitos nocivos da ansiedade e dor em pacientes em VM. Fizeram parte do grupo-controle 30 pacientes, e 30 do grupo que sofreu intervenção, no qual ambos passaram $90 \mathrm{~min}$ com o fone de ouvido, sem som e com música relaxante, respectivamente. Para mensurar a dor, foi utilizada a escala visual analógica da dor (EVA), aplicada no início, após $30 \mathrm{~min}$, 60 min e em 90 min de intervenção e, por último, 30 min após a intervenção. No início da intervenção, o resultado entre os grupos foi semelhante, porém o grupo que sofreu intervenção teve significativa diminuição da dor nas fases seguintes, levando a inferir que a música é um recurso não farmacológico que pode ser utilizado em pacientes em VM com objetivo de redução da dor.

Finalizando os tipos de condutas complementares e integrativas que a literatura traz como possíveis de serem aplicadas por fisioterapeutas, vem a aromaterapia. O estudo de Cho et al. (2013) mostrou benefícios de 10 inspirações de pedras contendo uma mistura de óleos essenciais de lavanda, camomila romana e néroli para a qualidade do sono e redução da ansiedade. Das técnicas utilizadas, esta é a menos convencional na conduta de fisioterapeutas e talvez fosse repassada para a equipe de psicologia ou de enfermagem, porém não exclui a possibilidade de utilização pelo fisioterapeuta após a finalização de seu atendimento, para garantir maior relaxamento e bem-estar durante as horas em que o paciente fica ocioso.

Independente do tipo de UTI estudada, a maior parte dos estudos focaram em realização de massagens para a redução de dor e sinais de ansiedade, contribuindo para a redução do uso de medicações como tramadol, morfina e fluoxetina, reduzindo custos no atendimento e o risco de futura dependência medicamentosa (MISTRALETTI et al., 2008; HU et al., 2015). Os demais benefícios estudados estão mais voltados a sintomas secundários, como constipação e problemas de sono. No entanto, estes também causam alterações de humor e aumentam o limiar de dor. Estes resultados validam a utilização de técnicas complementares no combate a dor e ansiedade, bem como os previne, a partir da redução de sintomas secundários que podem gerar quadros álgicos e de ansiedade (NASCIMENTO et al., 2012).

Apesar de nenhum estudo levantar os riscos relacionados à aplicação destas técnicas, devido à marginalização e pouca evidência destas, é necessária a realização de sua análise, além da comprovação de seus benefícios, já supracitados, para garantir segurança à equipe, aos pacientes e seus familiares.

\section{Considerações Finais}

A utilização de terapias complementares e integrativas no ambiente da UTI ainda é controversa; porém, evidências na literatura apontam seus benefícios e a possibilidade de utilização pela equipe de fisioterapia, principalmente na redução de dor e ansiedade, mas também na melhora da motilidade gastrointestinal, qualidade do sono e manutenção dos sinais vitais em valores basais. 
Apesar disso, novos estudos são necessários para gerar a possibilidade de meta-análises e maior segurança na afirmação dos benefícios destas técnicas.

\section{REFERÊNCIAS}

BRASIL. Ministério da Saúde. SIHSUS - Caderno de Informações de Saúde. Brasil. Brasília, 2010. Disponível em: http://datasus.saude.gov.br/sistemas-e-aplicativos/hospitalares/sihsus.

CHIASSON, A. M.; BALDWIN, A. L.; MCLAUGHLIN, C.; COOK, P.; SETHI, G. The effect of live spontaneous harp music on patients in the intensive care unit. Evidence-Based Complementary and Alternative Medicine, v. 2013, 2013.

CHO, M. Y.; MIN, E. S.; HUR, M. H.; LEE, M. S. Effects of aromatherapy on the anxiety, vital signs, and sleep quality of percutaneous coronary intervention patients in intensive care units. Evidence-Based Complementary and Alternative Medicine, v. 2013, 2013.

DEHGHAN, M.; FATEHI POOR, A.; MEHDIPOOR, R.; AHMADINEJAD, M. Does abdominal massage improve gastrointestinal functions of intensive care patients with an endotracheal tube?: a randomized clinical trial. Complementary therapies in clinical practice, v. 30, p. 122-128, 2018.

FUENTE-MARTOS, C.; ROJAS-AMEZCUA, M.; GÓMEZ-ESPEJO, M. R.; LARA-AGUAYO, P.; MORÁN-FERNANDEZ, E.; AGUILAR-ALONSO, E. Humanization in healthcare arises from the need for a holistic approach to illness. Medicina Intensiva (English Edition), v. 44, n. 2, p. 99-109, 2018.

HARVEY-WALKER, S. Can complementary and alternative therapies play a role in the emergency department?. Australian Emergency Nursing Journal, v. 1, n. 5, p. 29-32, 1998.

HU, R. F.; JIANG, X. Y.; CHEN, J.; ZENG, Z.; CHEN, X. Y.; LI, Y.; HUINING, X.; EVANS, D. J. W.; WANG, S. Non-pharmacological interventions for sleep promotion in the intensive care unit. Cochrane Database of Systematic Reviews, n. 10, p. 1-99, 2015.

JESUS, F. S; PAIM, D. M.; BRITO, J. O.; BARROS, I. A.; NOGUEIRA, T. B.; MARTINEZ, B. P.; PIRES, T. Q. Declínio da mobilidade dos pacientes internados em unidade de terapia intensiva. Revista Brasileira de Terapia Intensiva, v. 28, n. 2, p. 114-119, 2016.

MENDES, E. W.; PROCIANOY, R. S. Massage therapy reduces hospital stay and occurrence of lateonset sepsis in very preterm neonates. Journal of Perinatology, v. 28, n. 12, p. 815, 2008.

MIOKOVIC, T.; ARMBRECHT, G.; GAST, U.; RAWER, R.; ROTH, H. J.; RUNGE, M.; FELSENBERG, D.; BELAVÝ, D. L. Muscle atrophy, pain, and damage in bed rest reduced by resistive (vibration) exercise. Medicine and science in sports and exercise, v. 46, n. 8, p. 15061516, 2014.

MISTRALETTI, G.; CARLONI, E.; CIGADA, M.; ZAMBRELLI, E.; TAVERNA, M.; SABBATICI, G.; OMBRELLO, M.; ELIA, G.; DESTREBECG, A. L. L; IAPICHINO, G. Sleep and delirium in the intensive care unit. Minerva anestesiologica, v. 74, n. 6, p. 329-334, 2008.

MORAN, C. A.; CACHO, R. O.; CACHO, E. W. A.; SOUZA, K. G.; JOUZA, J. C.; FONSECA FILHO, G. G.; PEREIRA, S. A. Use of music during physical therapy intervention in a neonatal intensive care unit: a randomized controlled trial. Journal of Human Growth and Development, v. 25, n. 2, p. 177-181, 2015. 
NASCIMENTO, L. B.; SOUZA, V. P.; VERAS FILHO, J.; ARAÚJO, E. C.; SILVA, T. C. L. Integrative and complementary therapy in nursing: therapeutic touch in intensive care unit. Journal of Nursing UFPE, v. 6, n. 1, 2012.

ORWELIUS, L.; NORDLUND, A.; NORDLUND, P.; EDÉLL-GUSTAFSSON, U.; SJÖBERG F. Prevalence of sleep disturbances and long-term reduced health-related quality of life after critical care: a prospective multicenter cohort study. Critical Care, v. 12, n. 4, p. R97, 2008.

SAADATMAND, V.; REJEH, N.; HERAVI-KARIMOOI, M.; TADRISI, S. D.; VAISMORADI, M.; JORDAN, S. Effects of natural sounds on pain: A randomized controlled trial with patients receiving mechanical ventilation support. Pain Management Nursing, v. 16, n. 4, p. 483-492, 2015.

SILVA, T. A.; SCHUJMANN, D. S.; SILVEIRA, L. T. Y.; CAROMANO, F. A.; CAROLINA, F. Effect of therapeutic Swedish massage on anxiety level and vital signs of Intensive Care Unit patients. Journal of bodywork and movement therapies, v. 21, n. 3, p. 565-568, 2017.

WANG, A.; SUNDT, T.; CUTSHALL, S.; BAUER, B. Massage therapy after cardiac surgery.

Seminars in Thoracic and Cardiovascular Surgery, v. 22, n. 3, p. 225-229, WB Saunders, 2010. 
